\title{
Seismic inference of differential rotation in Procyon A
}

\author{
A. Bonanno ${ }^{1}$, M. Küker ${ }^{2}$, and L. Paternò ${ }^{3}$ \\ 1 INAF - Osservatorio Astrofisico di Catania, via S. Sofia 78, 95123 Catania, Italy \\ e-mail: abo@oact.inaf.it \\ 2 Astrophysikalisches Institut Potsdam, An der Sternwarte 16, 14482 Potsdam, Germany \\ 3 Dipartimento di Fisica e Astronomia, Sezione Astrofisica, Università di Catania, via S. Sofia 78, 95123 Catania, Italy
}

Received 23 June 2006 / Accepted 2 October 2006

\section{ABSTRACT}

\begin{abstract}
The differential rotation of the F5V-IV star Procyon A is computed for a class of models that are consistent with recent astrometric and asteroseismic data. The rotation pattern is determined by solving the Reynolds equation for motion, including the convective energy transport, which is anisotropic owing to the Coriolis force action that produces a horizontal temperature gradient between the poles and the equator. All the models show a decrease in the rotation rate with increasing radius and solar-type isorotation surfaces with the equator rotating faster than the poles, the horizontal rotational shear being much smaller for models with a less extended convective envelope. The meridional flow circulation can be either clockwise or counter-clockwise, and in some cases a double latitudinal cell appears. The rotational splittings are calculated for low-degree $p$-modes with $l=1, m=1$, and $l=2, m=1,2$; and it is shown that, for modes with $m=1$, the stronger the horizontal differential rotation shear, the weaker the effect on the average rotational splitting expected, whilst the opposite happens for the mode with $m=2$. On the basis of the present study, a resolution of $10 \mathrm{nHz}$ in individual oscillation frequencies seems to be necessary to test the different dynamical behaviour of the proposed models, which barely appears achievable even in the forthcoming space missions. However, the average over several observed splittings could produce the required accuracy.
\end{abstract}

Key words. stars: interiors - stars: evolution - stars: rotation - stars: oscillations - stars: individual: Procyon A

\section{Introduction}

One of the brightest stars in the sky $\left(m_{V}=0.34\right)$ is Procyon A ( $\alpha \mathrm{CMi})$, hereinafter simply called Procyon. It is an extensively studied object whose evolutionary phase is believed to be that of a subgiant star with a convective core, a radiative envelope, and a thin-surface convective zone, where $p$-modes are presumably excited (Christensen-Dalsgaard \& Frandsen 1983; Houdek et al. 1999).

For these reasons Procyon is a favourite target for asteroseismic observations. The first clear evidence for the presence of oscillations was obtained by Martić et al. (1999), but when it was observed in 2004 by the MOST space mission, the reported precision from the photometric measurements $(15 \mathrm{ppm})$ was not sufficient for detecting $p$-modes (Mattews et al. 2004). On the other hand, three different teams then reported the identification of $p$-modes on Procyon (Martić et al. 2004; Eggenberger et al. 2004; Claudi et al. 2005), leading to determining the large frequency separation in the range $54-56 \mu \mathrm{Hz}$. It is thus conceivable that future observation campaigns from ground or space will further refine the determination of the basic asteroseismic parameters and, in addition, will be able to measure a possible rotational splitting in this slow rotator.

The present work aims to determine the differential rotation for a class of models of Procyon, which are consistent with the presently available astrometric and asteroseismic data and with the related rotational splittings. As the evolutionary models of Procyon show that the mass in the surface convection zone is very small, ranging from $\sim 10^{-7} M_{\odot}$ to $\sim 10^{-4} M_{\odot}$ during the evolution, it is not clear which type (if any) of non-uniform rotation is present. In particular, the question we would like to investigate is whether a significant amount of latitudinal shear is present, so that a solar type rotation, $\Omega \sim \Omega(r, \theta)$, is generated, or instead $\Omega \sim \Omega(r)$, as is often assumed in asteroseismic investigations (Di Mauro et al. 2000).

Recent advances in the mean-field theory have proven to be very successful in explaining internal solar rotation. The solar rotation pattern can be explained in terms of angular momentum transport by the gas flow in the convection zone. Assuming that Reynolds stresses are the only relevant mechanism, Küker et al. (1993) found a remarkably good agreement between the results of their model and the "observed" rotation, as deduced from the inversion of rotational splittings. Kitchatinov \& Rüdiger (1995) and Küker \& Stix (2001) present a refined model that includes both the meridional flow and the convective heat transport and show that the flows driven by differential rotation and a small horizontal temperature gradient have opposite directions, owing to the anisotropic convective heat transport, and roughly balance each other in the solar convection zone. This analysis was extended to a generic class of zero-age main-sequence $\mathrm{F}$ stars of mass $M=1.2 M_{\odot}$ in which the convection zone was about $20 \%$ of the stellar radius (Küker \& Rüdiger 2005a) leading to the conclusion that a solar-type rotation is the most common rotational law, at least for moderately rotating solar type stars.

In the case of Procyon, one instead deals with an $M \sim$ $1.42-1.5 M_{\odot}$ evolved F star which presumably is ending or has just ended the main sequence phase, with a convection zone much shallower than in the cases discussed in Küker \& Rüdiger (2005a). 
Table 1. Characteristic quantities of the four Procyon models, where Model 1 includes diffusion of only H and He, and Models 2-4 include diffusion of all the elements. The suffixes ph and cz indicate the top (photosphere) and bottom of convective envelope, respectively.

\begin{tabular}{ccccccccccc}
\hline \hline Model & $M / M_{\odot}$ & Age (Gy) & $\log L / L_{\odot}$ & $\log R / R_{\odot}$ & $\log T_{\mathrm{e}}$ & $Z_{\mathrm{ph}}$ & $Y_{\mathrm{ph}}$ & $R_{\mathrm{cz}} / R_{\mathrm{s}}$ & $\Delta v_{0}(\mu \mathrm{Hz})$ & $\eta$ \\
\hline 1 & 1.42 & 2.2 & 0.829 & 0.315 & 3.812 & 0.01805 & 0.237 & 0.856 & 54.1 & 0.1 \\
2 & 1.45 & 2.1 & 0.830 & 0.315 & 3.810 & 0.01828 & 0.241 & 0.867 & 54.5 & 0.1 \\
3 & 1.48 & 1.8 & 0.839 & 0.310 & 3.816 & 0.01845 & 0.215 & 0.918 & 55.4 & 0.1 \\
4 & 1.50 & 1.9 & 0.847 & 0.310 & 3.816 & 0.01798 & 0.228 & 0.919 & 55.0 & 0.1 \\
\hline
\end{tabular}

\section{The evolutionary models}

The starting step of our investigation was to construct evolutionary models of Procyon by means of the GARSOM code (Schlattl et al. 1997, 1999; Bonanno et al. 2002) whose structure provides the basic stratification for solving the Reynolds equation (Küker \& Stix 2001).

In the present calculations, the OPAL opacities (Iglesias \& Rogers 1996) were used as implemented in the low-temperature regime by Alexander \& Fergusson (1994) tables. For the equation of state, we used either the OPAL-EOS 1996 (Rogers et al. 1996) or the MHD (Hummer \& Mihalas 1988; Mihalas et al. 1988; Däppen et al. 1988) tables. The OPAL-EOS 1996 tables have been updated by a relativistic treatment of the electrons and by improving the activity expansion method for repulsive interactions (Rogers \& Nayfonov 2002). The nuclear reaction rates were taken either from Bahcall et al. (1995) or Adelberger et al. (1998). The convection was treated by the classical mixing length theory. Microscopic diffusion of hydrogen, helium, and all the major metals was taken into account. The outer boundary conditions were determined by assuming an Eddington gray atmosphere.

The most recent determinations of the astrometric parameters of the visual binary orbit of Procyon were obtained by means of multichannel astrometric photometer observations during the period 1986-2004. The inferred mass of the primary component is found to be $M=1.43 \pm 0.034 M_{\odot}$ (Gatewood \& Han 2006), whilst a previous determination by Girard et al. (2000) reported $M=1.497 \pm 0.037 M_{\odot}$, with a parallax of $\Pi=283.2 \pm 1.5$ mas. Although the two measurements are consistent within the error bars, if only the Wide Field and Planetary Camera 2 observations are used in combination with the Hipparcos parallax, $\Pi=$ $285.93 \pm 0.88$ mas, the resulting mass is $M=1.42 \pm 0.04 M_{\odot}$, which is much closer to the Gatewood \& Han (2006) value. Here we assume the conservative range $1.42-1.50 M_{\odot}$ to constrain the mass of Procyon in our models, although the average value of these measurements would give a mass $M=1.45 \pm 0.02 M_{\odot}$.

The luminosity was estimated from the mean visual magnitude obtained by Allende Prieto et al. (2001) and bolometric correction by Flower (1996), so that $\log L / L_{\odot}=0.84 \pm 0.02$. Recent measurements of the angular diameter by Kervella et al. (2004) lead to $R / R_{\odot}=2.048 \pm 0.025$, while the parallax of Girard et al. (2000) gives $R / R_{\odot}=2.067 \pm 0.028$. The effective temperature has been calculated by Fuhrmann et al. (1997) who found $\log T_{\mathrm{e}}=3.815 \pm 0.006$.

The metallicity of Procyon, as derived from spectroscopic measurements, is close to the solar one (Takeda et al. 1996; Kato et al. 1996), and for simplicity we adopted the same (standard) metallicity mixture as for the Sun (Grevesse et al. 1996) so that at the surface $(Z / X)_{\mathrm{ph}}=0.0245$. The inclusion of metal diffusion in evolutionary models of Procyon leads to an almost complete depletion of the metal content at the surface (Chaboyer et al. 1994). The reason is that the gravitational settling is expected to take place below the convection zone that is only $\sim 10^{-4} M_{\odot}$ thick. In order to avoid this problem, we considered a mass loss that accords with the semi-empirical Reimers (1975) prescription, $\dot{M}=-4 \times 10^{-13} \eta(L / g R)$, where the surface gravity, $g$, luminosity, $L$, and radius, $R$, are expressed in solar units, and $\eta$ is an efficiency parameter, whose value depends on the evolutionary phase of the star from the main sequence to the red giant branch; here a value close to 0.1 turns out to be adequate.

The rotational velocity, as estimated by Allende Prieto et al. (2002), is $v \sin i=2.7 \pm 1.0 \mathrm{~km} \mathrm{~s}^{-1}$. If we assume that the rotational axis of Procyon is perpendicular to the plane of visual orbit ( $i=31.1^{\circ} \pm 0.6^{\circ}$, Girard et al. 2000), the surface rotational velocity should be $5.2 \pm 1.9 \mathrm{~km} \mathrm{~s}^{-1}$, which corresponds to a rotational period of about $20 \mathrm{~d}$. On the other hand, the rotational period is about $33 \mathrm{~d}$ in the extreme case of $i=90^{\circ}$.

Present asteroseismic data are not accurate enough to put strong constraints on the computed models, although several groups have succeeded in measuring the large frequency separation $\Delta v_{0}$. Martić et al. (2004) find $\Delta v_{0}=53.6 \pm 0.5 \mu \mathrm{Hz}$, while Eggenberger et al. (2004) find $\Delta v_{0}=55.5 \pm 0.5 \mu \mathrm{Hz}$. More recent measurements, obtained with the high-resolution spectrograph SARG operating with the $3.5 \mathrm{~m}$ Italian Galileo Telescope on the Canary Islands, instead report $\Delta v_{0}=56.2 \pm 0.5 \mu \mathrm{Hz}$ (Claudi et al. 2005).

We thus constructed evolutionary models of Procyon constrained by $54 \mu \mathrm{Hz} \leq \Delta v_{0} \leq 56 \mu \mathrm{Hz}$, and computed four representative models with masses in the range 1.42-1.5 $M_{\odot}$. In all the models, the the mixing length parameter, initial helium and metal abundances, diffusion of the elements, and the mass loss parameter $\eta$ have been adjusted in order to reproduce the observed luminosity and effective temperature within a box delimiting their determination uncertainties, with the abovementioned constraint on $\Delta v_{0}$. Exploration in the range $0.05 \leq$ $\eta \leq 0.15$ of the variation of the mass loss parameter $\eta$, which is still consistent with the asteroseismic requirements of matching the value of $\Delta v_{0}$, does not produce noticeable effects on the stratification of the convection zone and, by consequence, on its dynamical behaviour.

The basic characteristics of the four representative models are summarised in Table 1, while the evolutionary tracks of the models are presented in Fig. 1. Note that Model 2 represents the model with a mass corresponding to the average mass of the most recent determination, as already discussed.

\section{Differential rotation and heat transport in the outer convection zone}

The solar differential rotation was successfully reproduced by means of a mean-field model based on the Reynolds stress transport of angular momentum (Küker et al. 1993), improved by the inclusion of meridional flow and turbulent heat transport (Kitchatinov \& Rüdiger 1995). The inclusion of the abovementioned ingredients turned out to be crucial in obtaining results in agreement with the isorotation surfaces deduced from helioseismology, as the Taylor-Proudman theorem predicts 


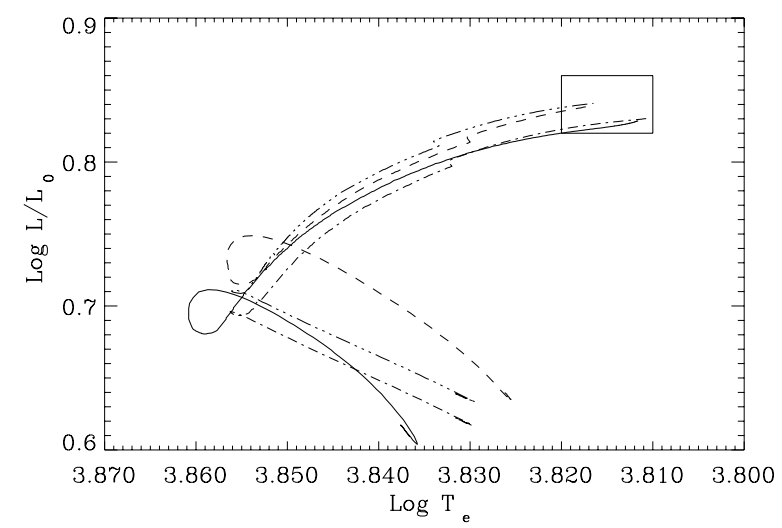

Fig. 1. The evolutionary tracks of the models summarised in Table 1. The solid line is for Model 1, the dashed for Model 2, the dot-dashed for Model 3, and the dot-dot-dashed for Model 4. The box indicates the uncertainties in the observation determinations of luminosity and effective temperature.

cylindrical surfaces of isorotation in an isothermal fluid at high Taylor numbers. Since the Taylor number assumes values as high as $10^{8}$ in the lower part of the solar convection zone, owing to its inviscid character, the state of rotation in cylinders would have been unavoidable (Rüdiger et al. 1998). The model has been applied to several types of stars, either fully convective or with outer convection zones (Kitchatinov \& Rüdiger 1999; Küker \& Stix 2001; Küker \& Rüdiger 2005a,b).

A relevant quantity for understanding the role of the turbulent transport of the angular momentum is the Coriolis number $\Omega^{*}=2 \tau \Omega$, where $\Omega$ is the stellar angular velocity and $\tau$ the characteristic turnover time of convective eddies (Rüdiger 1989). The radial dependence of $\Omega^{*}$ in the outer layers of the star for a representative model of Procyon is shown in Fig. 2. Since the inhomogeneous $\Lambda$-effect depends on $\Omega^{*-1}$ while the anisotropic $\Lambda$-effect depends on $\Omega^{*-3}$, it is then clear that they are both essential for correctly describing the turbulent transport of angular momentum (Kitchatinov \& Rüdiger 1995, 1999).

\subsection{Reference model}

Since the influence of rotation and large-scale meridional flow on the structure of the star is very small, we treat them as perturbations of an unperturbed, non-rotating, spherically symmetric reference state, which is a stellar convection zone in hydrostatic equilibrium, as computed by the stellar evolution code described above. In order to compute the differential rotation, we simplify the reference state by assuming a perfect gas with constant specific entropy and total heat flux throughout the convection zone. The differential equations for temperature and gravity (Poisson equation for a spherically symmetric gravitational potential with $g(r)=-\mathrm{d} \Phi / \mathrm{d} r)$ are those described in Kitchatinov \& Rüdiger (1995) for an analogous problem,

$$
\begin{aligned}
& \frac{\mathrm{d} T}{\mathrm{~d} r}=-\frac{g(r)}{c_{\mathrm{p}}} \\
& \frac{\mathrm{d} g(r)}{\mathrm{d} r}=-2 \frac{g(r)}{r}+4 \pi G \rho
\end{aligned}
$$

with the adiabatic relationship:

$\rho=\rho_{\mathrm{e}}\left(\frac{T}{T_{\mathrm{e}}}\right)^{1 /(\gamma-1)}$.

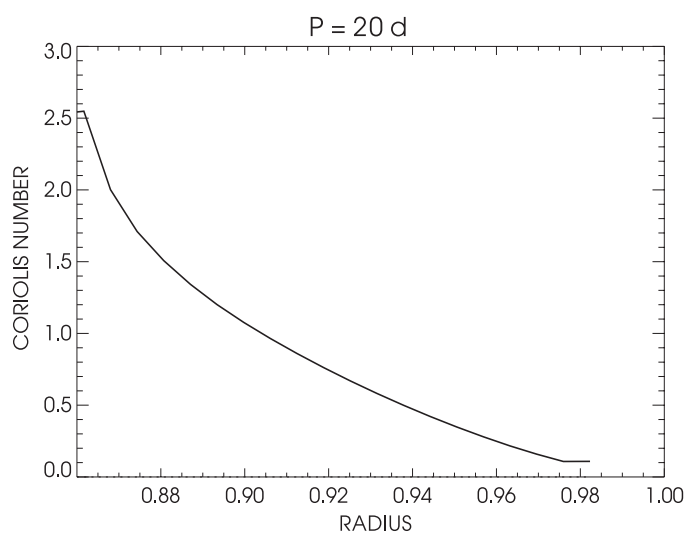

Fig. 2. Coriolis number as a function of the stellar fractional radius for a star with $M=1.45 M_{\odot}$ in the subgiant phase for a rotation period of $20 \mathrm{~d}$.

Here $c_{\mathrm{p}}=\mathcal{R} / \nabla_{\mathrm{ad}}$ is the specific heat at constant pressure, with $\mathcal{R}$ the gas constant and $\nabla_{\mathrm{ad}}$ the adiabatic gradient, $g(r)$ the gravity, $\gamma=\left(1-\nabla_{\mathrm{ad}}\right)^{-1}$ the adiabatic exponent, and the suffix $e$ indicates the values of the quantities at the outer boundary. The solution is determined by choosing a reference point from the model produced by the stellar evolution code, which provides the initial values necessary for the integration. Equations are then integrated outwards and inwards from the starting point at which the values from the reference model have been chosen, the inward integration being terminated at the point where the radiative flux reaches the total heat flux, which coincides with the bottom of the convection zone, and the outward integration terminated at the stellar radius as determined by the reference model. Thus Eqs. (1) and (2) constitute an initial value problem. To adjust the depth of the convection zone to the value of the reference model, we take the adiabatic gradient as the input parameter. For fully ionized monatomic gas, $\nabla_{\mathrm{ad}}=0.4$. In the outer parts of the convection zones, however, it is smaller owing to the partial ionization. The stratifications found by the stellar evolution code for the models of Procyon we considered show values of $\nabla_{\mathrm{ad}}$ as low as 0.1 in the outer parts of the convection zone. Since we assumed it constant with radius in our simple model, the values of $\nabla_{\mathrm{ad}}$ that give the best fit to the evolutionary models lie around 0.35 . We thus produce a convection zone with adiabatic stratification that closely resembles the one reproduced by the evolutionary models, which is used as the unperturbed reference state for the computation of the differential rotation. In spite of the simplifications used, the deviations of our adiabatic stratification from that of the evolutionary model are within a few percent in the relevant quantities everywhere in the convection zone.

\subsection{Heat transport}

Following the procedure described in Rüdiger \& Hollerbach (2004), the mean-field equation of convective heat transport is given by the following expression:

$\rho T \frac{\partial \bar{s}}{\partial t}=-\nabla \cdot\left(\boldsymbol{F}^{\mathrm{conv}}+\boldsymbol{F}^{\mathrm{rad}}\right)+\rho c_{\mathrm{p}} \overline{\boldsymbol{u}} \cdot \boldsymbol{\beta}$,

where $\bar{s}$ is the mean specific entropy. The transport terms on the RHS of Eq. (4) are the convective heat flux, $F_{i}^{\text {conv }}=\rho c_{\mathrm{p}}\left\langle u_{i}^{\prime} T^{\prime}\right\rangle=$ $\rho c_{\mathrm{p}} \chi_{i j} \beta_{j}$, the radiative heat flux, $F_{i}^{\mathrm{rad}}=-16 \sigma T^{3} \nabla_{i} T / 3 \kappa \rho$, and the heat advection by meridional flow, with $\overline{\boldsymbol{u}}$ the mean velocity field, where $\kappa$ is the opacity, $\sigma$ the Stefan-Boltzmann constant, and $\boldsymbol{\beta}=\boldsymbol{g} / c_{\mathrm{p}}-\boldsymbol{\nabla} T=-\nabla \delta T$ the super-adiabatic gradient. As the 
reference state has constant entropy $s_{0}$, we define the perturbation as $\delta s=\bar{s}-s_{0}$, and, since all derivatives of $s_{0}$ vanish, we can rewrite Eq. (4) in terms of $\delta s$ :

$\rho T \frac{\partial \delta s}{\partial t}=-\nabla \cdot\left(\boldsymbol{F}^{\mathrm{conv}}+\boldsymbol{F}^{\mathrm{rad}}\right)+\rho c_{\mathrm{p}} \overline{\boldsymbol{u}} \cdot \boldsymbol{\beta}$.

On applying the mass conservation law, $\nabla \cdot(\rho \overline{\boldsymbol{u}})=0$, replacing the temperature perturbation with the entropy perturbation, $\delta s=$ $c_{\mathrm{p}} \delta T / T$, we can rewrite the last term in Eq. (5) as $\rho c_{\mathrm{p}} \overline{\boldsymbol{u}} \cdot \boldsymbol{\beta}=$ $-\nabla \cdot(\rho T \overline{\boldsymbol{u}} \delta s)$. Since we find in some cases that the heat transport by the meridional flow exceeds that of the convective flux, we introduce a horizontally-averaged entropy perturbation:

$\delta \bar{s}_{1}=\frac{1}{2} \int_{0}^{\pi} \delta s \sin \theta \mathrm{d} \theta$

thus splitting $\delta s$ into the two terms $\delta \bar{s}_{1}(r)$ and $\delta s_{2}(r, \theta)$. We then drop $\delta \bar{s}_{1}$ from the advection term to prevent convective instabilities in our system and to obtain the equation of heat transport in its final form:

$\rho T \frac{\partial \delta s}{\partial t}=-\nabla \cdot\left(\boldsymbol{F}^{\mathrm{conv}}+\boldsymbol{F}^{\mathrm{rad}}+\rho T \overline{\boldsymbol{u}} \delta s_{2}\right)$.

\subsection{Equation of motion}

The equation of motion for the mean velocity field $\overline{\boldsymbol{u}}$ is (cf. Rüdiger 1989)

$\rho\left[\frac{\partial \overline{\boldsymbol{u}}}{\partial t}+(\overline{\boldsymbol{u}} \cdot \nabla) \overline{\boldsymbol{u}}\right]=-\nabla \cdot \rho Q-\nabla P+\rho \boldsymbol{g}$

where $Q$ is the one-point correlation tensor of fluctuating velocities $Q_{i j}=\left\langle u_{i}^{\prime} u_{j}^{\prime}\right\rangle$. On assuming axial symmetry, the azimuthal component of Eq. (7) can be written as

$\rho r^{2} \sin ^{2} \theta \frac{\partial \Omega}{\partial t}+\nabla \cdot t=0$

where $\boldsymbol{t}=r \sin \theta\left(\rho r \sin \theta \Omega \overline{\boldsymbol{u}}^{\mathrm{m}}+\rho\left\langle u_{\phi}^{\prime} \boldsymbol{u}^{\prime}\right\rangle\right)$. The two remaining components of Eq. (7) describe the meridional circulation $\overline{\boldsymbol{u}}^{\mathrm{m}}$, and can be represented by a single scalar function, the stream function $A$ :

$\overline{\boldsymbol{u}}^{\mathrm{m}}=\left(\frac{1}{\rho r^{2} \sin \theta} \frac{\partial A}{\partial \theta},-\frac{1}{\rho r \sin \theta} \frac{\partial A}{\partial r}, 0\right)$.

On taking the azimuthal component of the curl of Eq. (7), we obtain

$$
\begin{aligned}
\frac{\partial \omega}{\partial t}= & -\left[\nabla \times \frac{1}{\rho} \nabla(\rho Q)\right]_{\phi}+r \sin \theta \frac{\partial \Omega^{2}}{\partial z} \\
& +\frac{1}{\rho^{2}}(\nabla \rho \times \nabla p)_{\phi}+\ldots
\end{aligned}
$$

where $\omega=(\nabla \times \overline{\boldsymbol{u}})_{\phi}$ is the azimuthal component of the mean vorticity, $\partial / \partial z=\cos \theta \cdot \partial / \partial r-\sin \theta / r \cdot \partial / \partial \theta$ represents the gradient along the axis of rotation, and dots indicate second-order terms of minor importance. As the reference state is in hydrostatic equilibrium, $\boldsymbol{\nabla} P=-\boldsymbol{g} \rho$, we can rewrite the baroclinic term on the RHS of Eq. (10) in terms of specific entropy:

$\frac{1}{\rho^{2}}(\nabla \rho \times \nabla P)_{\phi}=-\frac{1}{c_{\mathrm{p}} \rho}(\nabla s \times \nabla P)_{\phi} \approx-\frac{g}{r c_{\mathrm{p}}} \frac{\partial s}{\partial \theta}$.

On neglecting the second-order terms, we have three terms on the RHS of Eq. (10); the first, which contains the Reynolds stresses, is purely viscous, while the second and third drive the meridional flow. Close to the poles, the gradient of the rotation rate along the rotation axis dominates, as the horizontal entropy gradient vanishes there. At low latitudes, on the other hand, $\partial \Omega / \partial z$ vanishes and the baroclinic term dominates. As both terms can be either positive or negative, they should enhance or cancel each other. For solar-type stars it has been found that both terms balance each other in a wide range of rotation rates, leading to a slow meridional flow and a strong differential rotation (Küker \& Rüdiger 2005).

\subsection{Transport coefficients}

Instead of the standard mixing-length theory we use the expressions derived by Kitchatinov \& Rüdiger (1999) for the convective heat transport and Reynolds stresses. The heat conductivity tensor, $\chi_{i j}$, can be written as the product of a scalar diffusion coefficient, $\chi_{\mathrm{t}}$, and a dimensionless tensor, $\Phi_{i j}$, so that $\chi_{i j}=\chi_{\mathrm{t}} \Phi_{i j}$, where $\chi_{\mathrm{t}}=c_{\chi} \tau_{\mathrm{c}} g \alpha^{2} H_{\mathrm{p}}^{2}\left\langle\beta_{r}\right\rangle / 4 T$, with $\tau_{\mathrm{c}}$ the turbulent eddy correlation time, $\alpha$ the classical mixing-length parameter, $H_{\mathrm{p}}$ the pressure scale height, $c_{\chi}$ a dimensionless coefficient for heat transport, and the triangular brackets averages over $\theta$. The stress tensor has the general form $\mathcal{T}_{i j}=-\rho Q_{i j}$, with the correlation tensor:

$Q_{i j}=-\mathcal{N}_{i j k l} \frac{\partial \Omega_{k}}{\partial x_{l}}+\Lambda_{i j k} \Omega_{k}$.

The first term of the RHS vanishes for rigid rotation and represents an anisotropic viscosity; the second one, the $\Lambda$-effect, is non-zero for rigid rotation, thereby causing differential rotation unless balanced by the meridional flow. We write the viscosity tensor, $\mathcal{N}_{i j k l}=v_{\mathrm{t}} \Psi_{i j k l}$, in a similar way as the convective heat transport, namely $v_{\mathrm{t}}=c_{\nu} \tau_{\mathrm{c}} g \alpha^{2} H_{\mathrm{p}}^{2}\left\langle\beta_{r}\right\rangle / 4 T$, where $c_{\nu}$ is a dimensionless coefficient for viscous transport. The $\Lambda$-effect appears only in two components of the correlation tensor, and it can be written as follows:

$Q_{r \phi}^{\Lambda}=v_{\mathrm{t}} V \sin \theta \Omega, \quad V=V^{0}+V^{1} \sin ^{2} \theta$

$Q_{\theta \phi}^{\Lambda}=v_{\mathrm{t}} H \cos \theta \Omega, \quad H=V^{1} \sin ^{2} \theta$.

The tensors $\Phi_{i j}$ and $\Psi_{i j k l}$ contain the anisotropy of the convective heat transport and the viscous part of the stress tensor, as computed by Kitchatinov et al. (1994). Together with the $\Lambda$-effect, coefficients $V^{0}$ and $V^{1}$ (Kitchatinov \& Rüdiger 1993, 2005) determine the transport of heat and angular momentum by convection. Note that the updated $\Lambda$-effect coefficients of Kitchatinov \& Rüdiger (2005) remove the discrepancy between theoretical and observed angular velocity distribution in the outer $30000 \mathrm{~km}$ of the solar convection zone found in models that use the older expressions. To adjust the model for the updated expressions for the $\Lambda$-effect, we now use $c_{v}=0.27$ with $\alpha=2$ rather than the values from Küker \& Rüdiger (2005a,b), who used $c_{v}=0.15$ with $\alpha=5 / 3$. With a Prandtl number, $\mathrm{P}_{\mathrm{r}}=v_{\mathrm{t}} / \chi_{\mathrm{t}}=0.8$ (Kitchainov et al. 1994), there is very good agreement between the computed and observed rotation pattern for the Sun. The transport coefficients have been calibrated with the Sun, whose differential rotation is known from helioseismology. Numerical simulations on the effect of changes of transport coefficients show that the strength of horizontal rotational shear is quite insensitive to changes in parameters, while meridional flow is more sensitive leading to slight changes in the rotational pattern. Both the existence of the $\Lambda$-effect and the anisotropy of the convective heat transport have been confirmed by simulations (Käpylä et al. 2004; Rüdiger et al. 2005). 


\subsection{Boundary conditions and gravity darkening}

The numerical code we used to solve Eqs. (6), (8), and (10) is the same as the one used by Küker \& Stix (2001). It uses a secondorder finite difference scheme that conserves angular momentum and energy. The equations for the reference model were solved by using a fourth-order Runge-Kutta scheme. In the equation of motion, we imposed stress-free boundary conditions on both boundaries. In the heat transport equation, we fixed the total luminosity on both boundaries, and assume that is independent of latitude, so that $L=4 \pi r^{2} F$. This fixes the radial component of the entropy gradient, but not the entropy itself, so does not preclude a horizontal entropy gradient. As we wrote the heat transport equation as a conservation law, these boundary conditions ensure that a stationary state is eventually reached.

Our code also includes the option of taking the gravity darkening into account by adding a rotation-dependent term to the heat flux equation:

$F=\frac{L}{4 \pi r^{2}}\left[1+\frac{2 \varepsilon}{3+\varepsilon}\left(\frac{3}{2} \cos ^{2} \theta-\frac{1}{2}\right)\right]$

where $\varepsilon=\Omega^{2} r^{3} / G M$ (Rüdiger \& Küker 2002). For a star like Procyon with a rotation period of $20 \mathrm{~d}$, a value of $\varepsilon=1.8 \times 10^{-4}$ is found, a low value that cannot affect luminosity. In fact we did not find a significant difference between runs with and without gravity darkening.

\section{Rotational splittings}

The rotational splittings of oscillation frequencies depend on the radial and latitudinal angular velocity distribution in the interior of the star. It could be of interest, essentially in the light of possible future observations, to calculate the rotational splittings for low-degree $p$-modes expected for our differential rotation models of Procyon. We used the following parameterization for the rotation law

$\Omega(r, \theta)=\sum_{i=0}^{i_{\max }} \Omega_{i}(r) \cos ^{2 i} \theta$,

so that the angular integration can be explicitly performed, and the rotational splittings are (Cuypers 1980)

$\delta v_{n l m}=\frac{m}{2 \pi} \sum_{i=0}^{i_{\max }} \int_{0}^{R} K_{n l m i}(r) \Omega_{i}(r) \mathrm{d} r$

with the kernels $K_{n l m i}($ Christensen-Dalsgaard 1997):

$$
\begin{aligned}
K_{n l m i}= & \rho r^{2}\left\{\left[\left(\xi_{r}-\xi_{h}\right)^{2}+\left(l(l+1)-2 i^{2}-3 i-2\right) \xi_{h}^{2}\right] Q_{l m i}\right. \\
& \left.+i(2 i-1) Q_{l m i-1}\right\} / \mathcal{I} .
\end{aligned}
$$

Here,

$\mathcal{I}=\int_{0}^{R} \rho r^{2}\left(\xi_{r}^{2}+l(l+1) \xi_{\mathrm{h}}^{2}\right) \mathrm{d} r$

and

$Q_{l m i}=\frac{2 l+1}{2} \frac{(l-|m|) !}{(l+|m|) !} \int_{-1}^{1} x^{2 i}\left[P_{l}^{m}(x)\right]^{2} \mathrm{~d} x$

$\xi_{\mathrm{r}}$ and $\xi_{\mathrm{h}}$ being the radial and horizontal components of the eigenfunctions, respectively. In our investigation the functions $\Omega_{i}(r)$ were determined from the numerical integration of the Reynolds Eq. (7) with $i_{\max }=4$.
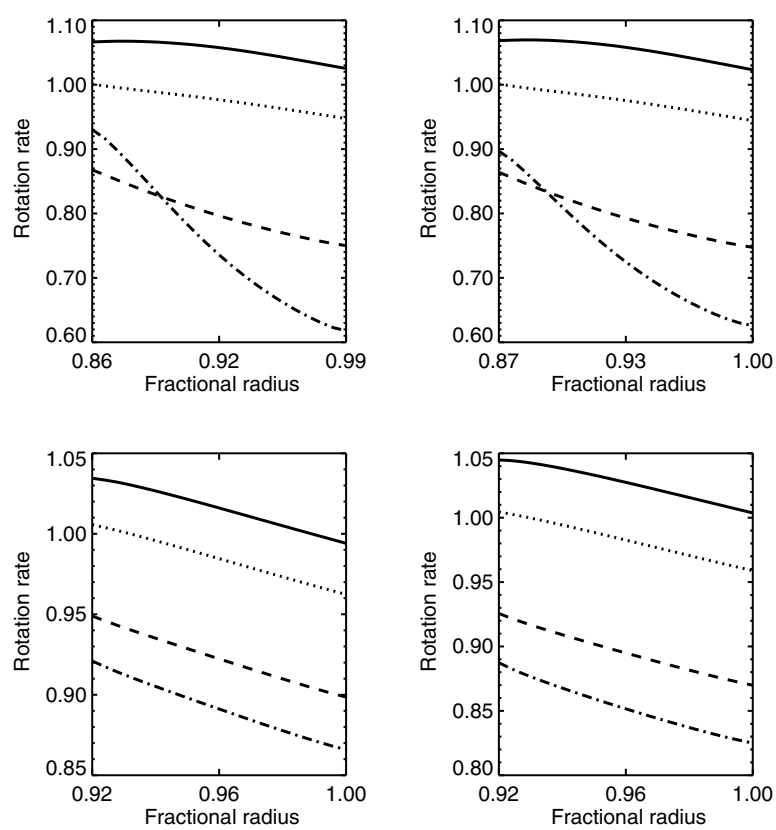

Fig. 3. The normalized angular velocity profiles for the four models with a rotation period $P_{\text {rot }}=20 \mathrm{~d}$. The four panels show: top left: Model 1; top right: Model 2; bottom left: Model 3; bottom right: Model 4. The different line-styles denote different latitudes: solid: equator; dotted: $30^{\circ}$; dashed: $60^{\circ}$; dash-dotted: poles. The angular velocity is normalized with respect to an average angular velocity $\bar{\Omega}$ as described in the text.

\section{Results}

The results of the present investigation are summarised in Figs. 3-7. Figure 3 describes, for each of the four computed Procyon models, the behaviour of the normalized rotation as a function of fractional radius at different latitudes, $\phi=0^{\circ}, 30^{\circ}, 60^{\circ}, 90^{\circ}$, for a rotation period $P_{\text {rot }}=20 \mathrm{~d}$. The angular velocity is normalized in terms of a weighting function based on the moment of inertia,

$\bar{\Omega}=\frac{\int \rho r^{4} \sin ^{3} \theta \Omega \mathrm{d} r \mathrm{~d} \theta}{\int \rho r^{4} \sin ^{3} \theta \mathrm{d} r \mathrm{~d} \theta}$

with the rotation period $P_{\text {rot }}=2 \pi / \bar{\Omega}$. Figure 4 describes, for the same models and rotation rate, the patterns of the meridional flow in the convection zone. Figures 5 and 6 show the same features of Figs. 3 and 4, but for a rotation period $P_{\text {rot }}=33 \mathrm{~d}$. Figure 7 shows the rotational splittings, as deduced from the four differential rotation models of Procyon for $P_{\text {rot }}=20 \mathrm{~d}$, calculated for the acoustic oscillation modes with $l=1, m=1$ and $l=2, m=1,2$.

In general, the overall characteristics of the distribution of angular velocity in all the models are of solar type, with an equatorial acceleration and the surface rotation essentially maintained throughout the whole convection zone.

The total horizontal shear at the surface, $\tilde{\Omega}=\left(\Omega_{\mathrm{eq}}-\Omega_{\mathrm{pole}}\right) / \bar{\Omega}$, ranges from 0.1 to 0.45 , depending on models and rotation period, which is approximately the same as the solar one, $\tilde{\Omega}_{\odot} \simeq$ 0.25 . The surface shear appears to be stronger in the higher rotation case $\left(P_{\text {rot }}=20 \mathrm{~d}\right)$. For Models 1 and 2, independently of their rotation rate, the rotation latitudinal shear is more pronounced at the surface than at the base of the convection zone, where there is a tendency to uniform rotation, as in the case of the Sun (Figs. 3 and 5). 

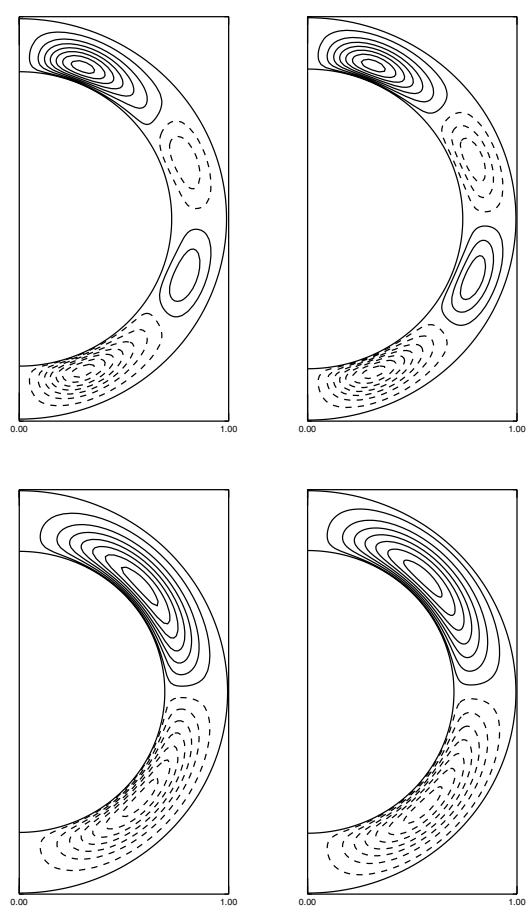

Fig. 4. The meridional flow patterns corresponding to the rotation profiles in Fig. 3. The four panels show: top left: Model 1; top right: Model 2; bottom left: Model 3; bottom right: Model 4. The solid lines of iso-velocity denote clockwise and the dashed ones counter-clockwise motions. For clarity, the depth of the convection zone has been enlarged in the diagrams; by a factor of 2 for Models 1 and 2 and 4 for Models 3 and 4 . The meridional motion maximum speeds at the stellar surface are: $100 \mathrm{~m} \mathrm{~s}^{-1}$ for Model 1; $87 \mathrm{~m} \mathrm{~s}^{-1}$ for Model 2; $41 \mathrm{~m} \mathrm{~s}^{-1}$ for Model 3; $37 \mathrm{~m} \mathrm{~s}^{-1}$ for Model 4
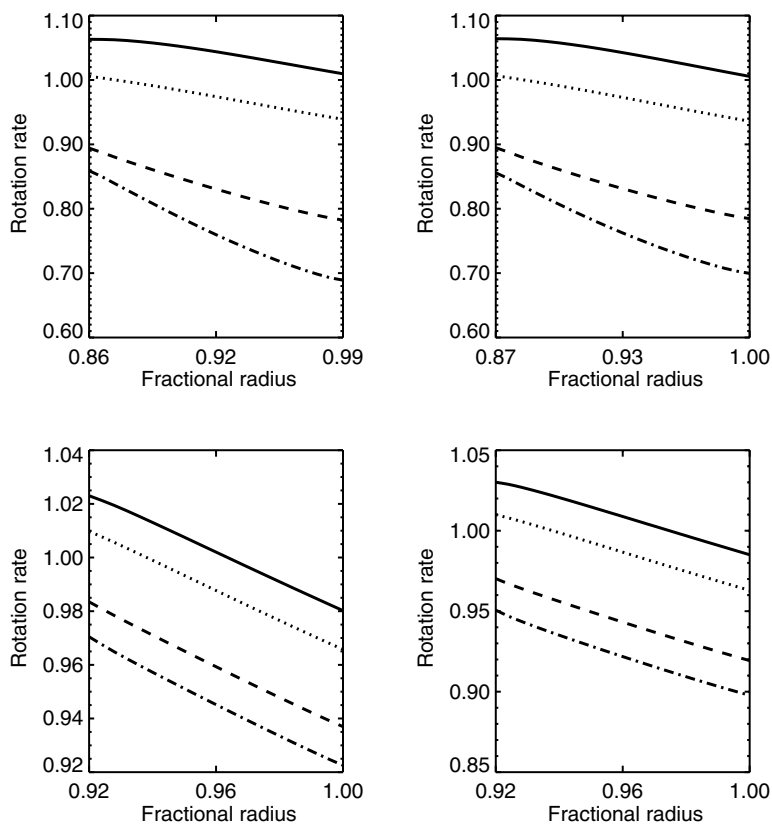

Fig. 5. The same as in Fig. 3 but for a rotation period $P_{\text {rot }}=33 \mathrm{~d}$.

The isorotation surfaces, not presented here, are practically the same for all the models and the two rotation rates considered, and they resemble the solar ones, nearly radial or slightly inclined with respect to the equatorial plane, but certainly not with a cylindrical shape, even in the lowest part of the convection zone. In most cases the meridional flow is in a single cell
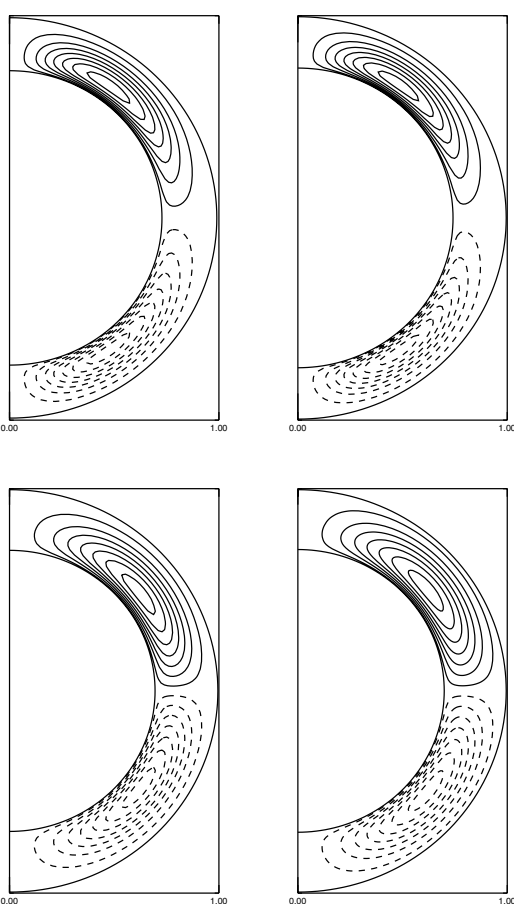

Fig. 6. The same as in Fig. 4 but for a rotation period $P_{\text {rot }}=33 \mathrm{~d}$. Here the meridional motion maximum speeds at the stellar surface are $65 \mathrm{~m} \mathrm{~s}^{-1}$ for Model 1, $57 \mathrm{~m} \mathrm{~s}^{-1}$ for Model 2, $17 \mathrm{~m} \mathrm{~s}^{-1}$ for Model 3, and $16 \mathrm{~m} \mathrm{~s}^{-1}$ for Model 4.
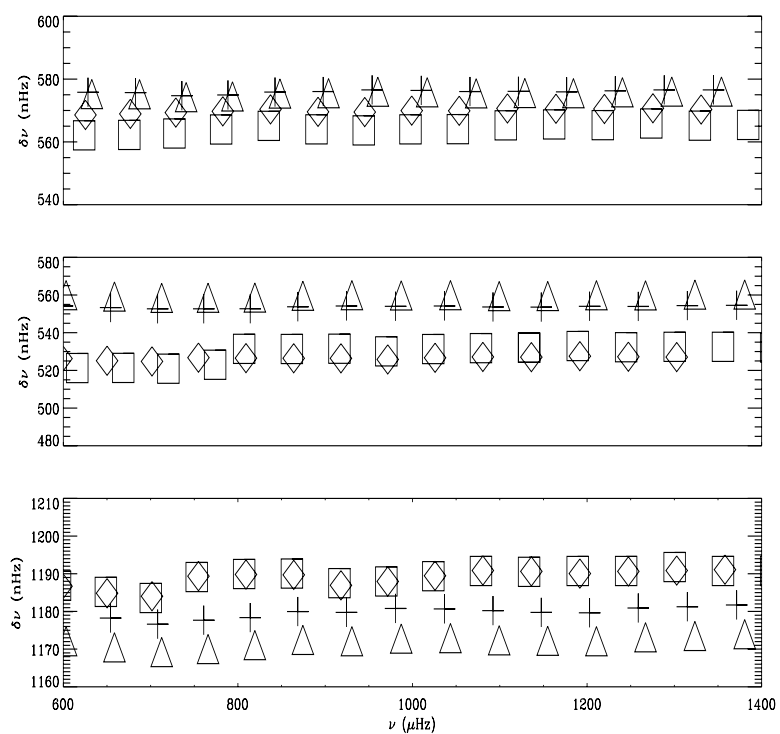

Fig. 7. The frequency rotational splittings of acoustic oscillation modes, $\delta v, l=1, m=1$ (top panel) $l=2, m=1$ (middle panel), and $l=2, m=$ 2 (bottom panel), as functions of the mode frequency, $v$, for the four models of differential rotation with a rotation period $P_{\text {rot }}=20 \mathrm{~d}$. The symbols identify the models: diamonds: Model 1; squares: Model 2; triangles: Model 3; crosses: Model 4.

extending from the poles to the equator, with the surface motion directed toward the equator, except for Models 1 and 2, at their higher rotation rate, where an additional small cell appears near the equator with the surface motion direct toward the pole (Fig. 4). The maximum speed of meridional motions at the surface ranges from $16 \mathrm{~m} \mathrm{~s}^{-1}$ to $100 \mathrm{~m} \mathrm{~s}^{-1}$, depending on the models and rotation period (Figs. 4 and 6). For comparison, the Sun's meridional flow at the surface is directed towards the poles with 
a speed of $\sim 10 \mathrm{~m} \mathrm{~s}^{-1}$ at intermediate latitudes, as deduced from Doppler-shift observations (Hathaway 1996).

The results for the rotational splittings essentially reflect the different dynamical behaviours of the Models 1 and 2, which have deeper convection zones and stronger horizontal rotational shears than Models 3 and 4, which have shallower convection zones and weaker horizontal rotational shears. For the modes with $m=1$ (top and middle panels of Fig. 7), there is a clear tendency of splittings related to the models with weaker rotational shear to approach the rigid rotation frequency of $579 \mathrm{nHz}$, corresponding to $P_{\text {rot }}=20 \mathrm{~d}$, while the splittings related to the models with stronger rotational shear are slightly smaller $(560-570 \mathrm{nHz})$, in the case of $l=1$, and smaller $(520-530 \mathrm{nHz})$, in the case of $l=2$, than the rigid rotation frequency. Opposite this is the case of modes with $l=2, m=2$ (bottom panel of Fig. 7) in which the splitting frequencies related to the models with weaker rotational shear appear to be smaller than those related to models with strong rotational shear. The differences in the splittings for Models 1, 2 and 3, 4 come mostly from the rotational profile, in particular from the latitudinal dependence of the shear. In fact, if the latitudinal dependence of the rotational law is artificially suppressed by setting $i_{\max }=0$ in Eq. (16), the differences in the splittings turn out to be much smaller.

As a general trend for all the models, the average frequency of splittings for the modes with $m=1(\overline{\delta v} / m \simeq 540-570 \mu \mathrm{Hz})$ is lower than the one for the modes with $m=2(\overline{\delta v} / m \simeq 590 \mu \mathrm{Hz})$. This depends mainly on the contributions of the angular function terms in kernels of Eq. (17), which are negative for $m=1$ and positive for $m=2$, and, by consequence, the sum in Eq. (17) is lowered in the first case and enhanced in the second case. This trend is especially evident for Models 1 and 2, which have a stronger latitudinal shear, in the case of $l=2$ modes $[\overline{\delta v} / m(m=1) \simeq 530 \mu \mathrm{Hz} ; \bar{\delta} v / m(m=2) \simeq 595 \mu \mathrm{Hz}]$.

\section{Conclusions}

Procyon has already been observed extensively and will presumably also be observed in the future with a greater accuracy both from the ground, by means of multi-site campaigns, or from space. Therefore more accurate determinations of the basic parameters of the star, as well as a higher resolution spectrum of its acoustic pulsations, are expected, which can be useful for verifying the results of the present investigation.

We have shown that a differential rotation of solar type can be maintained even in a very shallow convection zone and that its characteristics are sensitive enough to be able to distinguish among different stellar models; Models 1 and 2, which have a more extended convective envelope, show a larger latitudinal shear than Models 3 and 4, which have a less extended convective envelope. For the models with the higher rotation rate, the meridional motion at the lower latitudes changes from poleward for Models 1 and 2 to equatorward for Models 3 and 4. The features of the surfaces of isorotation seem to be adequate to ensure a possible cyclic dynamo activity in this star, whose surface manifestations could depend both on differential rotation and meridional flow.

Precise measurements of the rotational splittings should help tell the difference between models with deeper convection zones and strong latitudinal shears (Models 1 and 2) compared to those with shallower convection zones and weak latitudinal shears (Models 3 and 4). On the basis of the present study, a resolution of $\sim 10 \mathrm{nHz}$ in the oscillation spectrum seems to be necessary for testing our models of Procyon. Although this resolution is barely achievable for individual oscillation frequency measurements in the near future, even with the forthcoming space missions, since the mean value of the splitting for each mode is not strongly dependent on the frequency, the use of averages over several measured splittings could permit the required accuracy to be reached.

\section{References}

Adelberger, E. G., Austin, S. M., Bahcall, J. N., et al. 1998, Rev. Mod. Phys., 70, 1265

Alexander, D. R., \& Ferguson, J. W. 1994, ApJ, 437, 879

Allende Prieto, C., Asplund, M., García López, R. J., Lambert, D. L., \& Nordlung, A. 2001, in 11th Cambridge Workshop on Cool Stars, Stellar Systems and the Sun, ed. R. J. García López, R. Rebolo, \& M. R. Zapaterio Osorio, ASP Conf. Ser., 223, 760

Allende Prieto, C., Asplund, M., García López, R. J., \& Lambert, D. L. 2002, ApJ, 567, 544

Bahcall, J. N., Pinsonneault, M. H., \& Wasserburg, G. J. 1995, Rev. Mod. Phys., 67,781

Bonanno, A., Schlattl, H., \& Paternò, L. 2002, A\&A, 390, 1115

Chaboyer, B., \& Demarque, P. 1994, in 8th Cambridge Workshop on Cool Stars, Stellar Systems and the Sun, ed. J.-P. Caillault, ASP Conf. Ser., 64, 273

Christensen-Dalsgaard, J. 1998, Lecture Notes on Stellar Oscillations (Århus: Århus University)

Christensen-Dalsgaard, J., \& Frandsen, S. 1983, Sol. Phys., 82, 469

Claudi, R. U., Bonanno, A., Leccia, S., et al. 2005, A\&A, 429, L17

Cuypers, J. 1980, A\&A, 89, 207

Däppen, W., Mihalas, D., Hummer, D. G., \& Mihalas, B. W. 1988, ApJ, 332, 261

Di Mauro, M. P., Christensen-Dalsgaard, J., \& Weiss, A. 2000, in 3rd MONS Workshop, ed. T. C. Texeira, \& T. R. Bedding, Århus University, 151

Eggenberger, P., Carrier, F., Bauchy, F., \& Blecha, A. 2004, A\&A, 422, 247

Flower, P. J. 1996, ApJ, 469, 355

Fuhrmann, K., Pfeiffer, M., Frank, C., Reetz, J., \& Gehren, T. 1997, A\&A, 323, 909

Gatewood, G., \& Han, I. 2006, AJ, 131, 1015

Girard, T. M., Wu, H., Lee, J. T., et al. 2000, AJ, 119, 2428

Grevesse, N., Noels, A., \& Sauval, A. J. 1996, in 6th Annual October Astrophysics Conference in College Park, Maryland, ed. S. S. Holt, \& G. Sonneborn, ASP Conf. Ser., 99, 117

Hathaway, D. H. 1996, ApJ, 460, 1027

Houdek, G., Blamforth, N. J., Christensen-Dalsgaard, J., \& Gough, D. O. 1999, A\&A, 351, 582

Hummer, D. G., \& Mihalas, D. 1988, ApJ, 331, 794

Iglesias, C. A., \& Rogers, F. J. 1996, ApJ, 464, 943

Käpylä, P. J., Korpi, M. J., \& Tuominen, I. 2004, A\&A, 422, 793

Kato, K.-I., Watanabe, Y., \& Sadakane, K. 1996, PASJ, 48, 601

Kervella, P., Thévenin, F., Morel, P., et al. 2004, A\&A, 413, 251

Kitchatinov, L. L., \& Rüdiger, G. 1993, A\&A, 276, 96

Kitchatinov, L. L., \& Rüdiger, G. 1995, A\&A, 299, 446

Kitchatinov, L. L., \& Rüdiger, G. 1999, A\&A, 344, 911

Kitchatinov, L. L., \& Rüdiger, G. 2005, Astron. Nachr., 326, 379

Kitchatinov, L. L., Pipin, V. V., \& Rüdiger, G. 1994, Astron. Nachr., 315, 157

Küker, M., \& Rüdiger, G. 2005a, A\&A, 433, 1023

Küker, M., \& Rüdiger, G. 2005b, Astron. Nachr., 326, 265

Küker, M., \& Stix, M. 2001, A\&A, 366, 668

Küker, M., Rüdiger, G., \& Kitchatinov, L. L. 1993, A\&A, 279, L1

Martić, M., Schmitt, J., Lebrun, J.-C., et al. 1999, A\&A, 351, 993

Martić, M., Lebrun, J.-C., Appourchaux, T., \& Korzennik, S. G. 2004, A\&A, 418, 295

Matthews, J. M., Kushing, R., Guenther, D. B., et al. 2004, Nature, 430, 51

Mihalas, D., Däppen, W., \& Hummer, D. G. 1988, ApJ, 331, 815

Reimers, D. 1975, Mem. Roy. Soc. Sci. Liège, 8, 369

Rogers, F. J., \& Nayfonov, A. 2002, ApJ, 576, 1064

Rogers, F. J., Swenson, F. J., \& Iglesias, C. A. 1996, ApJ, 456, 902

Rüdiger, G. 1989, Differential Rotation and Stellar Convection: Sun and Solartype Stars (Berlin: Akademie-Verlag)

Rüdiger, G., \& Hollerbach, R. 2004, The Magnetic Universe: Geophysical and Astrophysical Dynamo Theory (Weinheim: Wiley-VCH Verlag)

Rüdiger, G., \& Küker, M. 2002, A\&A, 385, 308

Rüdiger, G., von Rekowski, B., Donahue, R. A., \& Baliunas, S. L. 1998, ApJ, 496, 691

Rüdiger, G., Egorov, P., Kitchatinov, L. L., \& Küker, M. 2005, A\&A, 431, 345

Schlattl, H., Weiss, A., \& Ludwig, H.-G. 1997, A\&A, 322, 646

Schlattl, H., Bonanno, \& Paternò, L. 1999, Phys. Rev. D, 60, 3002

Takeda, Y., Kato, K.-I., Watanabe, Y., \& Sadakane, K. 1996, PASJ, 48, 511 